\title{
Consideration on road transport vibration simulation techniques for packaging testing purposes
}

\author{
R. Pidl \\ Department of Applied Mechanics, Faculty of Mechanical Engineering \\ Information Technology and Electrical Engineering \\ Széchenyi István University \\ 9026 Győr 1 Egyetem tér, Hungary \\ pidlre@sze.hu
}

Abstract: Transportation is essential for many logistics systems and vibration is one of the most critic physical circumstances on road causing numerous possible damage sources. One of the primary sources of these damages is often the broadband random vibration. This effect cannot be handled with formal mechanical models; therefore, vibration test simulation is essential for packaging testing. The current vibration systems use Power Spectral Density (PSD) functions to control the intensity of random signal in laboratory based on Fast Fourier Transformation (FFT). Naturally, the PSD frequency does not contain time series data making it hard to determine how long a given simulation should be performed. In this paper, mathematical and probability methods are presented that can be used for representing real vibration circumstances.

Keywords: packaging, vibration, acceleration, PSD, simulation

\section{Introduction}

The movement of vehicle platform during traveling is a very complex phenomenon and depends significantly on the current state of the track-vehicle system. The productpackaging system has to be designed for vibration environment in order to avoid transport damages. For the time being, this is not completely resolved. For example, in Germany the amount of transport damages is between 200 and 250 million EUR based on insurance data [1]. Majority of these are caused by the not properly designed product-packagingcargo systems and their protecting functions, or during transport an extra effect hits the cargo on the platform, that could not be considered.

A non-adequate packaging could be a global problem causing millions of dollars of cargo damage, not counting the environmental effects. This way two separated options can be drawn. The first one is when the packaging is underestimated. The packaging cannot withstand the stress and the cargo is damaged, or even worse, when the nonadequate packaging causes accidents (also based on German insurance data: 12 death 
yearly are caused by non-adequate packaging design and cargo securing). This topic contains also transport safety issue. The other possibility is the overprotecting of the product. It is expensive, the vehicles are not utilized optimally, causing thereby more traffic and economic impact - not only from traffic but also from the waste of the additional and unnecessary packaging. The task of the engineer is therefore to optimize the protection of the product to prevent and avoid damages and to minimize the cost and the amount of packaging materials together.

Shocks, vibrations from the track-vehicle system can be one of the primer causes of damages. This is why it is of utmost importance to simulate these stresses before transportation as exactly as possible. For the time being no totally perfect solution is available for this issue [2]. Over the last 50 years, the vehicle vibration simulations developed from simple mechanic instruments to state-of-the-art computer controlled vibration tables [3]. But even the most advanced model considers the vehicle cargo platform as a fix body, where the fact is that those platforms can bend for prolonged time, also behave as a flexible body. It also increases to the complexity of the problem that besides the broadband frequency of movement- additional effects are present, namely prolonged and low frequency movements caused by braking, accelerating, etc. This effect is affected by the construction and is so complex that it cannot be fully modelled. The theoretic calculations, especially those without the use of computer, can have two degrees of freedom vibration system, whereas the platform is a vibration system with six degrees of freedom. Therefore, besides the theoretical calculations - that is simplified - laboratory simulations are also required for cargo planning [4]. The simulation of dynamic stress proposes many problems. It handles the platform as a fix body and cannot represent the movement correctly. With the use of the superposition principle, the real motion cannot be represented together with its longitudinal, vertical and lateral components [5]. The problem to be solved is what to choose as a relevant stress to be used for the protecting function design of the cargo [6].

\section{Currently used vibration simulation methods}

\subsection{Techniques}

Besides the standard procedures, a variety of alternative simulation methods also exist. In literature, 5 types of these methods can be identified [6] as follows:

- Time-based playback of recorded sign history

- Generating random vibration signal by normal distribution (Gauss simulation)

- Non-Gaussian distribution simulation:

○ non-stationarity simulation methods

$\circ$ transient event simulation

$\circ$ harmonic simulation

\subsection{Standard procedures}

The first equipment of transport simulation was quite simple: a table is moved with eccentric teeth, simulating large shocks, where the table and the teeth are not in constraint connection. The upper dead point is maximized. This means practically the current U.S. 
standard listed as ASTM D999-08 [7]. Repeating large shocks can be reproduced, but no real vibration effects could be simulated with it.

A better solution is the ASTM D3580 [8] and the corresponding ISO/IEC 60068-2-6 [9] standard vibration equipment with clean sinus acceleration signs. This equipment has hydraulic power transmission and the sinus vibration is performed in the frequency range of 1-100 Hz. The hydraulic actuator holds acceleration between $0.5-1 \mathrm{~g}$ in the whole frequency range with decreasing trajectory amplitude with the rising of frequency. These apparatuses are controlled with trajectory steering. No computer aided control is required; inner electronics can handle it simply. The rate of frequency change (sweep) can be controlled separately in octave/min according to the standard. Also, according to the standard, the sweeping speed is $0.5-5$ octave/min. The test has to be performed with upwards sweeping and after reaching the upper frequency limit, with downwards sweeping as well. Unfortunately, this standard is rarely used for the time being, despite the fact that the critic resonance stripes could be well determined with them. If random acceleration-time functions are considered on vehicle cargo platforms, it can be seen that they always contain harmonic sinus components. These components are coming from the not balanceable driven wheels and the actual rev of the engine. These harmonic components can cover a very broad frequency range, because they depend on the actual speed, the motor rev depending on accelerating and braking and the gear position, respectively. It must be noted here that diesel engine upper rev is much lower than Otto engines' upper rev, thus the $100 \mathrm{~Hz}$ upper frequency limit at diesel engines is excessive. If real transport environment were considered, the sinus sweeping should be parted to frequency ranges to simulate first gear, accelerating from $0-10 \mathrm{~km} / \mathrm{h}$, then second gear, speeding from neutral rev again, and so on, of course with the necessary deceleration and gear shift backs. This could be simulated with sine sweep with sweeping to a low upper frequency, then back to neutral rev and so on. In the packaging testing laboratory of the Széchenyi István University two types of electrodynamic vibration test machines are installed: MTS (Fig. 1.) and TIRA (Fig. 2.), where mentioned standards' tests above can be performed. 


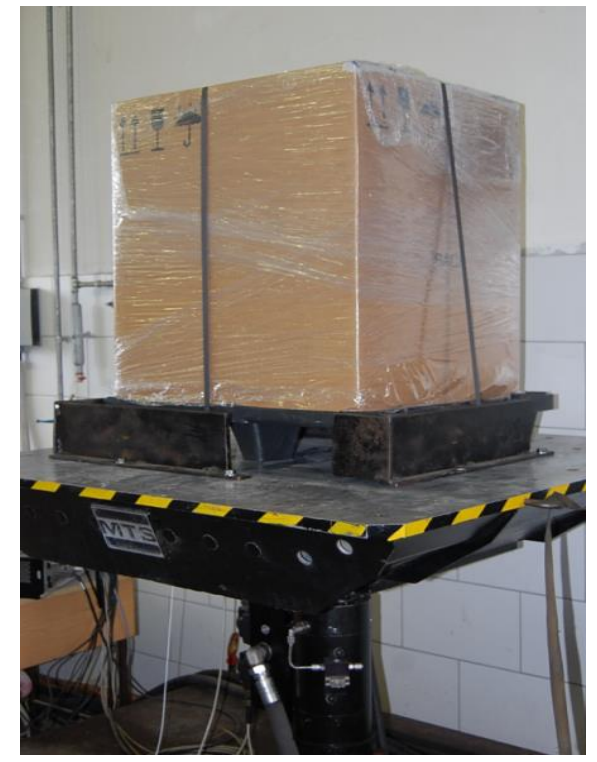

Figure 1. Servo-hydraulic vibration system

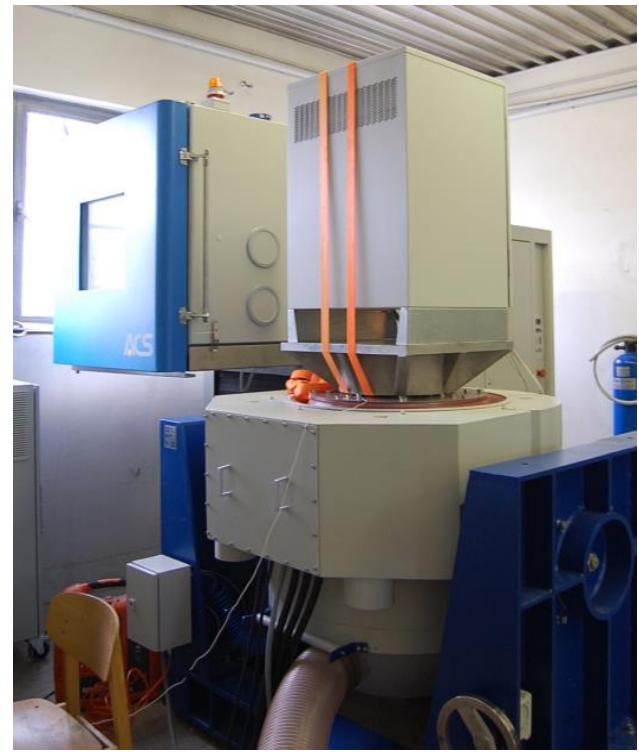

Figure 2. Electro-dynamic vibration system

It is obvious that the behaviour of the tested cargo is different in the frequency ranges during upward or downward sweeping. The explanation and reason for this phenomenon could be the late reaction of the test subject to various frequency changes, and in this instant the cargo moves away in reaction to the motion of the table and starts to bounce on the table. However, it must be stated here that the deterministic mechanical models typify the partly parallel, partly serial connected swinging parts built from springs and 
absorbers as a swing system connected to each other. The functions are created for the theoretical excitation excluding the fact that separation could occur both in the road-wheel and in the platform-cargo connection in the frequency ranges. At the moment of separation, the force-excited swing system starts to vibrate in its own frequency - that frequency differs from the original exciting frequency. That continues until the separated cargo hits the vibration table again. At this moment the two separate, independently vibrating swing systems meet and neither their amplitude nor their frequency and phase are the same. This is critic, hence the hits could damage the packed product too. The other critical part of the sinus sweeping is where the exciting frequency meets the frequency of the cargo. This is called resonance and here 6-8 times the exciting acceleration amplitude affects the test subject. It is very complicated to determine however, whether this frequency exists in a given transport vehicle, and if it exists, how often. This harmonic sinus vibration test should be performed for sure, but in my opinion the test procedure should be changed.

Many standards exist for broadband, random excited vibration tests (ASTM D4169-16, MIL-STD-810F, ISO 13375:2003) [11-13]. For the time being these broadband, random excited vibration tests are the most widespread. These could be performed with both electrohydraulic and electrodynamic vibration tables, with computer calculated PSDfrequency functions from Fast Fourier Transformation where the upper and lower frequency limits and the PSD and RMS values in the various frequency ranges are determined. These functions were created after many measurements on transport vehicles and recorded for various times of the acceleration-time function and determined the PSDfrequency function with FFT and after many measurements a statistically representative sample was created where normal distribution of the PSD values were presumed [14]. The standard PSD characteristics must be added per frequency range to the control computer of these equipment and this computer creates random transitional situations, but during the full run time all such situations are created. These equipment are very complex and expensive, because they have to manage the current swing trajectory and current acceleration furthermore the control unit has to determine the actual PSD value simultaneously. A further question is the required duration of the simulation depending on the transportation distance. This is a very important practical question, because especially in case of large distance transports- simulating the whole length is impossible and the PSD function will not excite real values but it will compress all the unique vibrations in the whole frequency range and the small bandwidth vibrations also [15]. It can be said that with the transforming of vibrations to frequency ranges the possibility of measuring in time range will be lost. Many testing laboratories realized this and the development of this method is in research focus for the time being. For the required test duration Basquin is suggesting the following model [6]:

$$
\frac{t_{j}}{t_{k}}=\left(\frac{a_{t}}{a_{j}}\right)^{k}
$$

where: $t_{j}$ is the current transport duration,

$t_{k}$ is the test duration,

$a_{t}$ is the test intensity, 
$a_{j}$ is the transport intensity,

$k \quad$ can be freely chosen between 2-5.

The simulation problems erecting from the PSD frequency functions can be analysed with understanding the FFT, that is, the complex problematic of transiting from timelines to frequency lines (Fig. 3.).

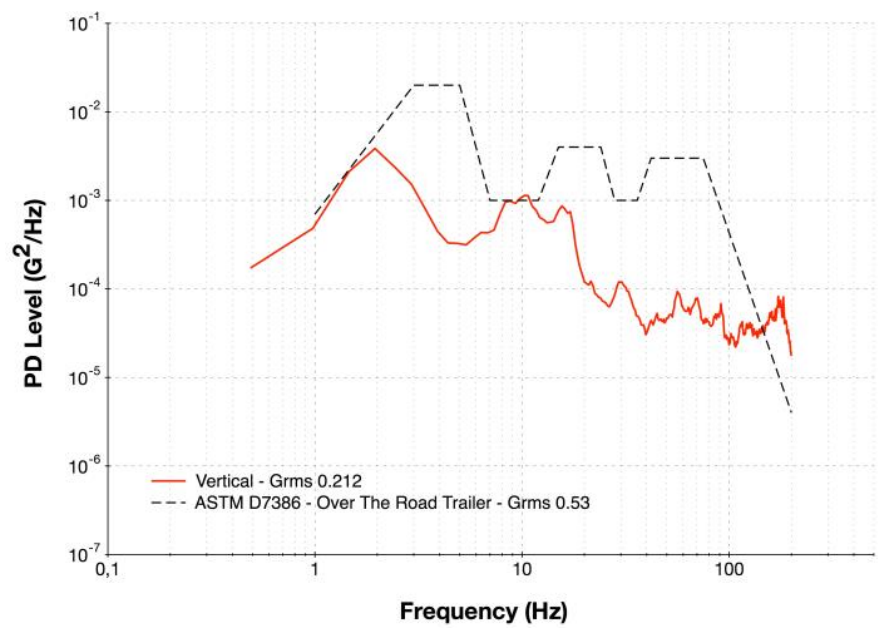

Figure 3. Field measured PSD frequency function compared to ASTM D4169-16 vibration standard method

\subsection{Non- standard procedures}

\subsubsection{Simple playback of real recorded acceleration-time function}

Computer controlled vibration machines offer the possibility to digitally record the acceleration-time function on a vehicle transporting a given cargo, then after input to the computer the machine can repeat it. The problem with this method that it is valid only for the given situation, other cargo or vehicle or driver or weather conditions could result in a completely different acceleration-time function. Furthermore, the test duration has to be the same as the transport duration. Because of these constrains, this method is not too widespread, but it has some advantages, for example, the transients during transport (road errors, railroad crossing, etc.) can also be recorded and used. Furthermore, the various transport environments (urban roads, motorways, industry roads) can be repeated according to the correct order.

\subsubsection{Non-stationary and transients to FFT simulation, Gaussian distribution simulation}

The FFT is one of the greatest inventions of mathematics from the last century [17]. It lowers the necessary operation time compared to discrete Fourier transformation, so this advantage should be used. But the Gaussian distribution used in FFT excludes the values outside the normal distribution at vibration tests. A method has to be found that includes 
the values outside the standard deviation thus bias the normal (Gaussian) distribution function according to our aim.

Obviously, it is possible to adjust the probability density function of normal distribution with various probability calculation or mathematic statistic methods in order to avoid the loss of high intensity signs (even losing one is a problem), and still maintaining the test simulation intensity at normal level. One possible solution is to bias the Gaussian curve with the kurtosis calculation method, shown in Fig. 4. It can be proven analytically that in normal distribution the kurtosis value is 3 , and if the kurtosis $>3$ it is called leptokurtic, if its equal to 3, mesokurtic and if kurtosis $<3$ it is called platykurtic, respectively. Hence normal distribution in the above case can be regarded as standard, the kurtosis values should be transformed by subtracting 3 from its value and measuring the excess kurtosis. This makes the kurtosis of normal distribution to zero, and all cases are either negative or positive.

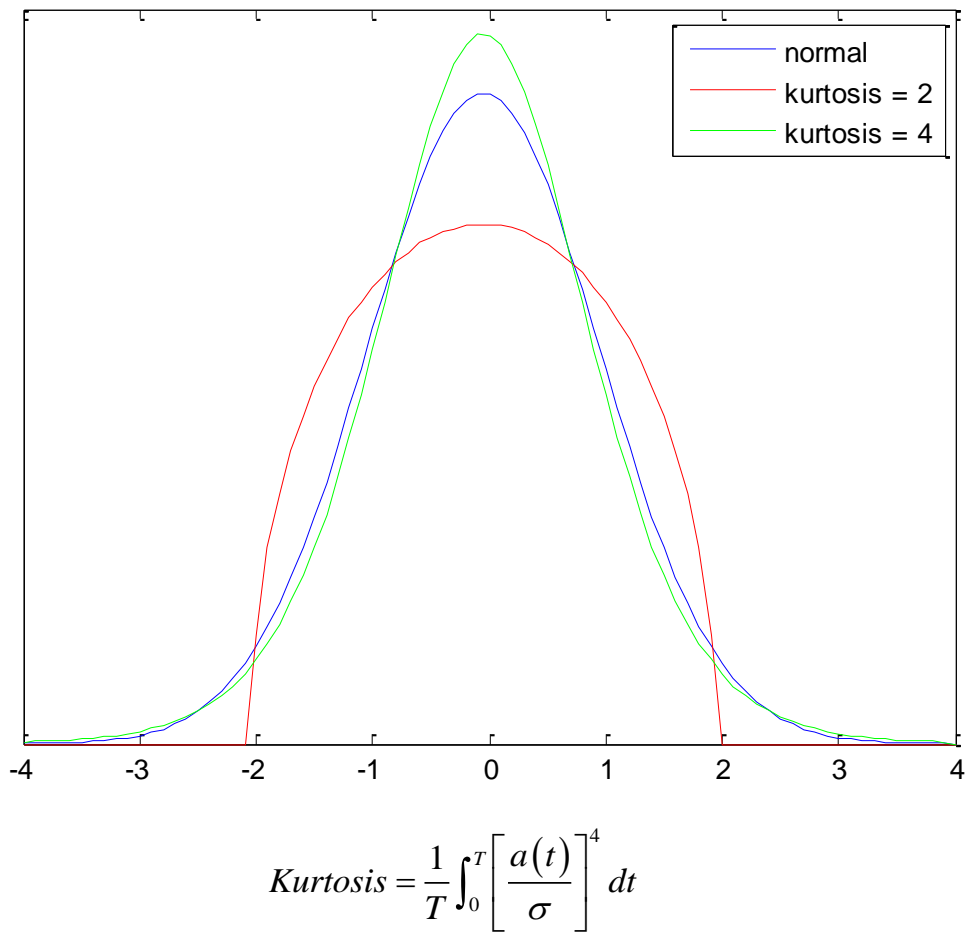

Figure 4. The effect of various kurtosis values on the Gaussian curve

A debate is going on that which kurtosis value recreates the most exactly the original acceleration-time function for various transport solutions. Even the with kurtosis adjusted normal distribution-like distribution does not contain the non-stationers of the transport processes. This non-stationarity comes from road surface quality - even the slightest change results in a completely different acceleration-time function. Also, a cause is the constant change in vehicle speed. Other speed ranges are used on motorways, urban roads, 
suburban roads, and all that affect the vibration during transport. Fig. 5/a. shows a field measured acceleration-time history in 40 seconds with the kurtosis value of 7 , while Fig. 5/b. shows an acceleration-time history using by vibration systems' random generator with value of 3 .

a)

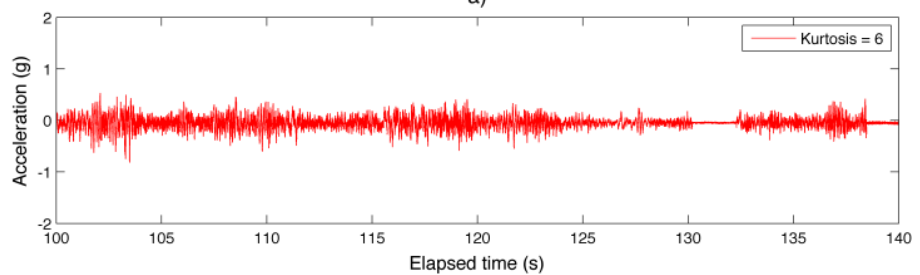

b)

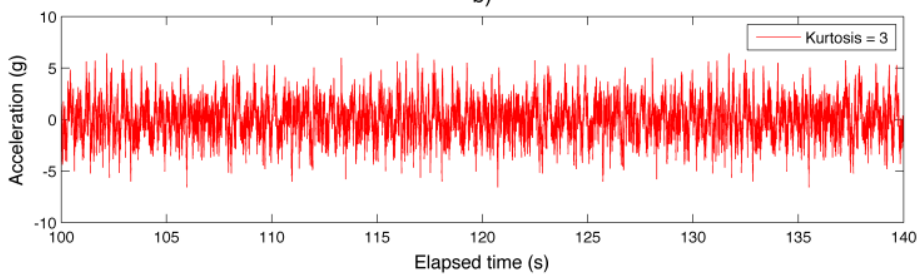

Figure 5. Acceleration-time history, a) field measured signal displaying road surface errors, b) random signal using by vibration controllers in laboratory

One possible solution to simulate road surface quality changes is to use leptokurtosis. Another possible solution for simulation non-stationarity could be the spectrum splitting, split the normal distribution to sequences, the Wavelet transformation and the use of Bayesian detection [19].

The major problem of the standard methods is that these exclude the transient events from the vibration profile, even though in practice the majority of cargo damages could be traced back to this unique, hard hit-type stresses [20]. The aim of the authors' doctoral thesis is a method for simulating these transient events on vibration equipment, and what type of acceleration-time functions those have in case of a railroad crossing or road surface damages. It has to be determined how many times these occur during a given transport and whether it should be included in the beginning or at the end of the simulation process. This leads to the packaging padding size decisions. It is without doubt that the viscoelastic characteristics of cushioning foams made from various polymer types are different, thus it has to be decided what the greater cargo damage causes: the big hit at the start of the test that annuls the elasticity of the foam letting the product vibrate freely or when the foam exhausted from the long vibration gets compacted and harder and hits the product with more excessive force than the not exhausted foam.

\section{Analytical model for testing the effects of random non-Gaussian vibrations}


Obviously, the vibration tests' purpose is to protect the goods after vibration stress. In the model we presume that the product is a solid body protected on its eight corners with cushioning material (usually a polymer foam), held together with a box. It may not be considered as the material of the box. This system is applied to the vibration machine table. If the product is a solid body it can be characterized with its weight and geometric dimensions. The cushioning material will be replaced with parallel connected $k$ spring constant and with $c$ damping ratio. For the analysis of the system, the spring and damping characteristics should be known. In first iteration the $\mathrm{k}$ and $\mathrm{c}$ are presumed to be linear, that is not true in practice of course, but it can be used to determine the own frequency of the system with a vertical shock.

The motion equation will be the following [20]:

$$
m(\ddot{u}+\ddot{z})+c \dot{u}+k u=0
$$

where: $m[\mathrm{~kg}] \quad$ is the product weight,

$k[\mathrm{~N} / \mathrm{mm}] \quad$ is the spring constant,

$c[\mathrm{~N} / \mathrm{mm}] \quad$ is the damping ratio,

$z$ [mm] is the total swing length of the vibration table (input),

$u=x-z[\mathrm{~mm}]$ is the motion of the product relative to the vibration table.

Parameter $\mathrm{u}$ can be calculated from the equation if $\mathrm{m} \mathrm{c} \mathrm{k}$ and $\mathrm{z}$ are known and then $\mathrm{x}$ can be calculated as $\ddot{x}=\ddot{u}+\ddot{z}$.

If such a system is dropped from a pre-defined height we can determine the peak acceleration with an accelerometer as the following:

$$
\ddot{x}_{\max }=\left|\frac{2 \zeta^{2}-1}{\sqrt{1-\zeta^{2}}}\right| \exp \left(\frac{-\zeta \Pi}{2 \sqrt{1-\zeta^{2}}}\right) v \omega
$$

where: $\zeta$ is the damping ratio,

$v$ is the vertical collision speed,

$\omega$ is the natural angular frequency.

If this test is performed on an actual package and the parameters are defined, the behaviour to vibration effects can be determined with numeric simulation. A possible simulation model is the MapleSim (Waterloo, Maple, Inc., Canada) software. This software is also capable of adjusting the Gaussian distribution PSD with kurtosis values 3,5 and 7 [13].

With transporting test of the actual package, during which the vibration acceleration values were measured on the product itself, performing an FFT on the resulting acceleration-time function it was clear that the numeric simulation could determine the effecting acceleration of the product with good iteration by using a kurtosis value of 7.2. 
That also means that by choosing 7.0 kurtosis value on the vibration table the original acceleration-time function can be reproduced.

\section{Summary}

During logistics processes, various external physical events affect the packed products. The product may tolerate these effects but excess external effects have to be handled by the protecting function of the sustainable packaging. From among these various external effects, this paper dealt with the random vibration occurred during transport and loading. In this paper first a review was performed about the currently used testing and calculation methodologies that are imperfect from many aspects. The formal models exclude even relevant effecting factors and laboratory testing cannot reproduce real world effects either. Of course, it has to be mentioned that real world effects also contain numerous random elements - question is when to consider something normal or above normal vibration effect. After many laboratory-testing methods had been chosen that could be regarded significant based on statistics analysis and after considering some safety factors, the real circumstances can be iterated well. The Gaussian distribution used for vibration testing simulation has to be altered. The best solution at hand is the use of Gaussian distribution with altered kurtosis. With altering kurtosis values a probability density function can be created that could reproduce the measured real function during laboratory testing quite well. This study also mentioned that the currently neglected harmonic excited vibration tests should be introduced in an altered form. With this method, the determination of the natural own frequency of the product-package system could be compared with the affecting vibration frequencies of transport and material handling and if these do not match, then additional protection is no needed. On the other hand, if in some frequency bands the functions match, either with the change of product construction or packaging damping system adjustment the damaging vibration effects could be minimized. At the end of the paper a possible calculation method was presented, that based on the above experiences could give a starting value for the characteristics of the cushioning material that could shorten the iteration time of the cushioning design process.

\section{References}

[1] Röbisch K: Transportsicherer profitieren 2014 von geringen Schadenquoten, IUMIKonferenz, 2015 Berlin

[2] Rouillard V, Sek M: Creating transport vibration simulation profiles from vehicle and road characteristics, Packaging Technology and Science 2013; Vol. 26, No. 2: pp. 82-95.

[3] Harris C, Piersol A: Harris' shock and vibration handbook, (2001).

[4] Böröcz P: Analysing the functions and expenses of logistics packaging systems, Proceedings of FIKUSZ 2009: Symposium for young researchers, Budapest, Hungary, 2009, pp. 29-39.

[5] Charles D: Derivation of environment descriptions and test severities from measured road transportation data, Journal of the IES 1993; Vol. 36, No. 1, pp. 37-42.

[6] Lepine J, Rouillard V, Sek M: Review on Road Vehicle Vibration Simulation for Packaging Testing Purposes, Packaging Technology and Science, 2015, DOI:10.1002/pts.2129, pp 672-682. 
[7] ASTM D999-08: Standard Test Methods for Vibration Testing of Shipping Containers, ASTM International: West Conshohocken, PA, 2008. DOI: 10.1520/D0999-08.

[8] ASTM D3580-95: Test Methods for Vibration (Vertical Linear Motion) Test of Products, ASTM Standard: West Conshohocken, PA, 2010; 4. DOI: 10.1520/D3580-95R10.

[9] IEC-60068-2-6: Environmental Testing - Part 2-6: Tests - Test Fc: Vibration (sinusoidal), International Electrotechnical Commission, 2007.

[10] Barbosa RS: Vehicle Vibration Response Subjected to Longwave Measured Pavement Irregularity, Journal of Mechanical Engineering and Automation, 2012, pp 17-24.

[11] ASTM D4169-12: Standard Practice for Performance Testing of Shipping Containers and Systems, ASTM International: West Conshohocken, PA, 2016, DOI: 10.1520/D4169-16

[12] MIL-STD-810 F: Environmental Test Methods and Engineering Guides. Department of Defense Test Method Standard, USA, 2000.

[13] BS EN ISO 13355:2003: Packaging, Complete, Filled Transport Packages and Unit Loads. Vertical Random Vibration Test, BSI: London, UK, 2002; 16.

[14] Böröcz P, Singh SP, Singh J: Evaluation of Distribution Environment in LTL Shipment between Central Europe and South Africa, Journal of Applied Packaging Research, Vol. 7, No. 2, 2015, Paper 3.

[15] Shires D: On the time compression (test acceleration) of broadband random vibration tests, Packaging Technology and Science 2011; Vol. 24, No. 2., pp. 75 87.

[16] Steinwolf A, Connon III WH: Limitations of the Fourier Transform for Describing Test Course Profiles, Sound and Vibration, 2005, pp. 12-17.

[17] Dongarra J, Sullivan F, Guest Editors' Introduction: The Top 10 Algorithms, Computing in Science \& Engineering, Vol. 2, No. 1, pp. 22-23, 2000, DOI:10.1109/MCISE.2000.814652

[18] Böröcz P, Vastag Gy: Good Vibrations: Lessons from Packaging for the Global Supply Chain.", POMS 2015, 1318.

[19] Böröcz P, Singh SP: Measurement and Analysis of Vibration Levels in Rail Transport in Central Europe, Packaging Technology and Science, 2016, Early View, DOI:10.1002/pts.2225

[20] Hosoyama A, Saito K, Nakajima T: Effectiveness of Stationary Non-Gaussian Random Vibration Testing and its Influence on Packaging, Proceedings of 26th IAPRI Symposium on Packaging 2013, pp 86-97. 